\title{
Optimal Algorithm Selection in Multimodal Medical Image Registration
}

\author{
Husein Elkeshreu ${ }^{1 \#}$ \& Otman Basir ${ }^{2}$ \\ ${ }^{1,2}$ Electrical and Computer Department, University of Waterloo, 200 University Ave W, Waterloo, \\ ON N2L 3G1, Canada.
}

\#corresponding author.

Type of Review: Peer Reviewed.

DOl: http://dx.doi.org/10.21013/jas.v15.n4.p1

\section{How to cite this paper:}

Elkeshreu, H., Basir, O. (2020). Optimal Algorithm Selection in Multimodal Medical Image Registration. IRA International Journal of Applied Sciences (ISSN 2455-4499), 15(4), 55-72. doi:http://dx.doi.org/10.21013/jas.v15.n4.p1

\section{(C) Institute of Research Advances.}

\section{(cc) EY-NO}

This work is licensed under a Creative Commons Attribution-Non Commercial 4.0 International License subject to a proper citation to the publication source of the work.

Disclaimer: The scholarly papers as reviewed and published by the Institute of Research Advances (IRA) are the views and opinions of their respective authors and are not the views or opinions of the IRA. The IRA disclaims of any harm or loss caused due to the published content to any party.

Institute of Research Advances is an institutional publisher member of Publishers International Linking Association Inc. (PILA-CrossRef), USA. The institute is an institutional signatory to the Budapest Open Access Initiative, Hungary advocating the open-access of scientific and scholarly knowledge. The Institute is a registered content provider under Open Access Initiative Protocol for Metadata Harvesting (OAI-PMH).

The journal is indexed \& included in CAS Source Index of Chemical Abstracts Service of American Chemical Society (USA), Index Copernicus (IC Value 85.27), WorldCat Discovery Service (USA), CrossRef Metadata Search (USA), WorldCat (USA), OCLC (USA), Open J-Gate (India), EZB (Germany) Scilit (Switzerland), Airiti (China), Bielefeld Academic Search Engine (BASE) of Bielefeld University, Germany, PKP Index of Simon Fraser University, Canada. 


\begin{abstract}
Many medical applications benefit from the diversity inherent in imaging technologies to obtain more reliable diagnoses and assessments. Typically, the images obtained from multiple sources are acquired at distinct times and from different viewpoints, rendering a multitude of challenges for the registration process. Furthermore, different areas of the human body require disparate registration functional capabilities and degrees of accuracy. Thus, the benefit attained from the image multiplicity hinges heavily on the imaging modalities employed as well as the accuracy of the alignment process. It is no surprise then that a wide range of registration techniques has emerged in the last two decades. Nevertheless, it is widely acknowledged that despite the many attempts, no registration technique has been able to deliver the required accuracy consistently under diverse operating conditions. This paper introduces a novel method for achieving multimodal medical image registration based on exploiting the complementary and competitive nature of the algorithmic approaches behind a wide range of registration techniques. First, a thorough investigation of a wide range of registration algorithms is conducted for the purpose of understanding and quantifying their registration capabilities as well as the influence of their control parameters. Subsequently, a supervised randomized machine learning strategy is proposed for selecting the best registration algorithm for a given registration instance, and for determining the optimal control parameters for such algorithm. Several experiments have been conducted to verify the capabilities of the proposed selection strategy with respect to registration reliability, accuracy, and robustness.
\end{abstract}

Keywords: computer system registration, multimodal medical image registration, medical images, visual registration, machine learning.

\title{
Introduction
}

Medical imaging is an indispensable tool in medical diagnosis and analysis [1]. In order to achieve accurate and comprehensive assessment multiple images are obtained using different imaging technologies, often at different acquisition times and from different viewpoints. To derive such an assessment, it is imperative that all images are registered to ensure that the extracted information relates to the target area of interest [2]. A few examples among the distinct types of medical imaging methods existing are Ultrasound images(US), Computed Tomography (CT), and Single-Photon Emission Computed Tomography (SPECT)[3]. Each one of these imaging modalities possesses different properties, rendering them capable of providing a spectrum of views and insights into the human body. For example, CT and MRI modalities are frequently utilized when highlighting issues related to the anatomical structure of the imaged area. In contrast, PET and SPECT modalities are employed to provide functional insights into the imaged area[4].

Typically, a registration algorithm comprises stages such as geometric transformation, alignment assessment function or cost function, and a control scheme to optimize the alignment process [5]. This paper introduces a novel method for achieving multimodal medical image registration based on exploiting the complementary and competitive nature of the algorithmic approaches behind a wide range of registration techniques. First, a thorough investigation of a wide spectrum of registration algorithms is conducted for the purpose of understanding and quantifying their registration capabilities as well as the influence of their control parameters. Subsequently, a supervised randomized machine learning strategy is proposed for selecting the best registration algorithm for a given registration instance, and for determining the optimal control parameters for such algorithm. Various optimization strategies are employed in medical image registration, including the steepest descent, Newton's methods, genetic algorithm (GA[6]. This paper aims to increase the intelligence of the system proposed by examining the effect of the optimization strategy on the registration algorithm's overall performance. The selection of an optimization strategy and the best registration algorithm plays an essential role in improving the accuracy, reliability, and robustness of the selected candidates.

A variety of existing multimodal registration algorithms have been proposed for medical image registration, each with its own technique for executing the registration process[7]. Current individual 
registration algorithms are categorized by their pros and cons that reflect their efficiency. For example, an individual registration algorithm can produce optimal results for a particular medical image while, at the same time, providing inferior results for others. Although on average, several algorithms have a superior effect, no specific algorithm has been identified as ideal for all datasets with regard to medical images[8]. It is, therefore, unclear how an optimum existing registration algorithm can be defined for a given dataset. Developing a new registration strategy has been the general trend in several existing research strands. However, although this might solve complications related to specific medical images, it cannot be employed universally and across fields of inquiry. The main issue then arises concerning the fact that an individual registration algorithm does not provide high performance on all the datasets one may wish to register. Furthermore, choosing the most suitable method is likely to enhance overall performance. Therefore, instead of finding a new registration algorithm, determining a novel strategy that can select the best registration algorithm and the best optimization method for an input dataset will increase reliability, accuracy, and robustness while decreasing the search time required. Thus, based on the problems outlined, it has become paramount to determine how to recognize the most appropriate current registration algorithm and optimization techniques for solving the issues at hand (rather than creating innovative registration algorithms). As a result, a universal registration system needs to be found that can produce an optimum result for all input datasets.

This paper, therefore, introduces a novel method for selecting the best registration algorithm and optimization algorithm, based on specific performance criteria, from a group of registration algorithms used for a variety of input datasets. This innovative method is based on the supervised machine learning technique where the dataset is labelled, and a roulette wheel technique is used to select the best registration strategy. The final solution is based on applying the labelling strategy to the input dataset, where labels are the registration algorithm (An), and an optimization strategy (Ок) leads to optimal performance, with ideal accuracy. To increase the intelligence of the proposed system, a new registration strategy was established, the aforementioned machine learning and roulette wheel selection method, that represents the registration algorithms and optimization strategy (Аn, Ок). Several experiments were then conducted, the results of which show that the novel approach leads to a considerably faster, reliable, accurate, and more robust registration strategy.

\section{Background and Literature Review}

There is an increasing interest in the utilization of image processing in techniques that supply the means of observing objects inside the human body. Progress in operative and precise image processing techniques have resulted from the development of computer technology; moreover, in the medical sphere, such advances are useful for research as well as for diagnosis, treatment, and planning[9]. Additionally, it is essential that such images be geometrically aligned in order to obtain information and improved observation. As such, many hospitals are increasingly applying computed tomography (CT) and functional modalities, as well as introducing magnetic resonance (MR) to achieve this end[10]. An example of this is the increasing frequency with which numerous methods are applied to image patients in the course of their diagnosis and for planning their treatment in the case of computed tomography (CT)[11].

Intermodal image registration can be a means of engaging with the problems related to the alignment of two images having different areas of view and different modalities, resolutions, and slice orientations. The expression 'image registration' is applied to indicate the mapping equivalent points' procedure in both modalities, known as spatial transformation[12]. During this process, two images, namely the target and the reference, are aligned into a single coordinate system. This enables the slight differences between the two to be observed. The following could be causes for the reference and target images to be different: (a) they were not taken at the same time; (b) they were taken using different devices, such as MRI, CT, SPECT and PET were utilized, and (c) in order to receive a two or three-dimensional image, they were 
taken from different viewpoints [3]. In the field of clinical diagnosis and treatment, there are numerous possible applications of such imaging techniques in treating various diseases such as cardiac, abdominal, pelvic, liver, retinal, renal, and tissue diseases[13]. The registration procedure is utilized by merging structural images, such as MRI or CT, or functional images, such as SPECT or PET, in various applications such as computer-aided surgery and diagnosis[14].

In order to acquire more extensive information concerning the patient, the combined data from different images is applied. This may include methods such as magnetic resonance imaging (MRI), and SPECT. Numerous methods are currently being applied to demonstrate image alignment registration assignment, which may be classified in accordance with a small number of standards[15]. The shifting technique is a new method applied to register medical images. During this procedure, one image is taken and slowly superimposed onto a second picture. Subsequently, each movement's similarity is evaluated and, finally, the highest similarity for both paradigms is selected [16]. Similarly, various procedures may be applied to classify different registration methods. A nine-dimensional technique, which provides high-quality categorization when analyzing medical images, is recommended by many researchers[17]. Table 1 [See Annexure I] outlines the development of numerous registration methods and the provision of several criteria used to categorize them. These are in accordance with various standards: the registration base (intensity- or feature-based)[18]; the dataset dimension (2D or 3D)[19]; the nature of the transformation (rigid or non-rigid); the transformation domain (global or local); the subject (intra-subject, inter-subject, or atlas subject); the modality (monomodal or multimodal); and the interaction (interactive, automatic, or semi-automatic)[17].

Since the medical images described in the following sections of this research typify the imaging applied for various purposes, they have been utilized for numerous registration experiments. Examples of the functional and anatomical classifications of the medical image are shown in Table 2 [See Annexure I].

\section{Algorithm Selection Problem}

The algorithm selection problem, which may be resolved comprehensively, concerns choosing the optimal algorithm for a particular problem. Researchers have been employing an enormous endeavor, during the past decade, to solve problems, not by formulating new algorithms, but by identifying existing ones. Therefore, the algorithm selection problem has become increasingly appropriate[20].

The fact that an individual registration algorithm cannot supply the optimal performance for every problem needing to be resolved has, for a considerable time, been accepted by researchers. They have also accepted that it is probable that the most appropriate method will improve general performance. Concerning the occurrence of problems, these researchers estimated the calculation time of LKH and Concorde, as did Sharma et al. in [21]. Moreover, Komal et al., by applying specific ranking methods in order to choose the optimal procedure according to ranking, claim to have resolved the selection problem[21]. Rice published the first interpretation of the algorithm selection. The rather simplistic primary kind summarized in this section is an area of process and a space of inputs that merge each inputalgorithm couple according to its outcome. When encountering a problem, this procedure may be applied in order to choose the most suitable algorithm [22]. The main steps applied for the resolution of algorithm selection problems are explained in the three-dimension diagram in Figure 1 [See Annexure I], where datasets space is $(D)$, registration algorithms space is $(A)$, and performance space is $(P)$.

The optimal algorithm which maximizes performance $(\mathrm{P})$ is required. It is necessary to find the optimal registration algorithm (a), with input dataset $(x)$, which maximizes $\mathrm{P}(\mathrm{a}(\mathrm{d}))$ as in the equation

$a=S(f(d)$

Where: 
$\mathrm{a} \in \mathrm{A}$, Registration Algorithm Space

$\mathrm{d} \in \mathrm{D}$, Data Space Space

$\mathrm{S}$, is Selection mapping

$\mathrm{P}$, Performance Space

\section{Problem Formulation}

The selection of a registration algorithm can be considered as an issue to be addressed in a threedimensional space. Figure 1 illustrates the $\mathrm{x}$-axis as a series of optimization strategies (Ок) and a series of registration algorithms $(\mathrm{An})$. In contrast, the y-axis defines the performance $(\mathrm{P})$ of the registration algorithms, with the z-axis denoting various multiple datasets $\left(\mathrm{D}_{\mathrm{j}}\right)$.

The optimal performance with dataset $\mathrm{d}_{3}$ is provided by the registration algorithm $\left(\mathrm{A}_{1}, \mathrm{O}_{4}\right)$, as illustrated in Figure 1. Consequently, divergent results will be obtained if dataset one is chosen and mapped to many registration algorithms, such as ( $\mathrm{An}, \mathrm{O}_{\mathrm{K}}$ ). Furthermore, Figures 2, 3 and 4 [See Annexure I] indicate that different results will be obtained again if the same process is applied to the $\mathrm{d} 2$ dataset. Finally, it should be recognized that maximum performance, in the case of all datasets, cannot be obtained from any superior registration algorithm; moreover, across all registration algorithms, no datasets can surpass all others. Therefore, the question arises of how to choose a registration strategy that will generate a highperformance outcome.

In the three-dimensional space, the information given is contained in datasets (D) and different registration algorithms with a different optimization strategy space (An, ОK), where the output is the performance $(\mathrm{P})$ of each input dataset with all registration algorithms and optimization strategies or vice versa. $\mathrm{P}$ is a function of (A, $\mathrm{O}$, and $\mathrm{D})$, and $\mathrm{S}($.$) is the selected function, as represented in equation (1).$ The selected registration algorithm $(\mathrm{An})$ and optimization strategy $\left(\mathrm{O}_{\kappa)}\right.$ provide the best value of $(\mathrm{P})$ with the dataset $\left(d_{j}\right)$.

$P=S\left(A_{n} O_{k} d_{j}\right)$

where

- $\mathrm{n}$ : is the number of registration algorithms such that $n=1,2,3 \ldots r$,

- $\mathrm{j}$ : is the size of the dataset such that $j=1, \ldots, m$.

- $\mathrm{k}$ : is the optimization algorithms number.

- $D$ : is the datasets such that $\mathrm{d}_{1}, \mathrm{~d}_{2}, \mathrm{~d}_{3}, \mathrm{~d}_{\mathrm{m}} \in \mathrm{D}$

- A: is the algorithm set such that $A_{1}, A_{2}, A_{3} \ldots A_{n} \in A$

- $\mathrm{O}$ : is the optimization strategy.

By increasing the recommended technique's intelligence in the former work, the objective of the problem statement is to enhance the technique used in the previous work [23]. Since the optimal outcome for every input dataset is not obtained from an individual registration algorithm, it was intended to recognize the current most effective algorithm, and not to enhance new algorithms in order to resolve the registration challenge. It was aimed to examine how a broad scope of registration algorithms performed within several situations and modalities. The purpose of this was to develop an optimization strategy with the ability to address a series of registration circumstances and an automatic technique that can optimize the choice of a registration algorithm.

Figure 5 [See Annexure I] depicts the automatic method, which is a multimodal medical image registration selection technique. The input comprises a dataset space (D), an optimization strategy space (An, Ок) and a registration algorithm, and the output is the optimal registration algorithm. 
Therefore, the selection of the ideal optimization strategy and the optimal registration algorithm is an algorithm selection problem. Three essential steps apply in any registration algorithm, namely, optimization strategy, cost function and transformation; moreover, these motivate the selection. It would be achievable to obtain the best of both options and to enhance the general performance if it would be possible to recognize the optimal registration algorithm and optimization strategy.

A supervised machine learning method forms the basis of the suggested innovative technique, in which the dataset, in conjunction with a roulette wheel method, is labelled according to the optimization strategy $\left(\mathrm{O}_{\mathrm{K}}\right)$ and the registration algorithm (An.). The training dataset label is represented by the optimization strategy and the registration algorithms (Ок, An) in order to enhance the suggested technique's intelligence. Subsequently, in order to generate a learning paradigm, the labelled training dataset is utilized. The new learning paradigm can supply additional primary objectives for new datasets. Furthermore, the output, in association with the precise anticipated output, can be assessed by the learning paradigm algorithm, the difference being the error, to enable it to make an appropriate adjustment to the paradigm. Figure 6 [See Annexure I] shows that a labelling method for assessing the performance of optimization strategies and dataset-based registration algorithms is included in the research portrayed in this essay.

The labelling method comprises three steps:

1- Dataset Space: a series of benchmark datasets (a pair of medical images)

2- Registration Algorithm Space: a series of registration algorithms

3- Optimization Algorithm Space: a series of different optimization strategies

4- Performance Measure: An accuracy evaluation, which is utilized as a performance standard for the most significant degree of precision, is attained by applying an optimization strategy and a registration algorithm for a specific dataset.

Tests were conducted on every registration algorithm within the algorithm space combined with the following four optimization strategies: Powell's method [7], simulated annealing (S.A.), Newton's methods [4], and the steepest descent. This was in order to choose the optimization and algorithm strategies (OK, An), which supply the highest performance standard (Figure 6) [See Annexure I]. Several experiments in data labelling, according to the number of datasets, were conducted at the initial stage, involving the testing of three registration algorithms with four different optimization algorithms (Figure 6), being 12 classifications per dataset (Table 3 ).

Therefore, the registration algorithm and optimization algorithm that provides the highest accuracy with the input dataset is selected as a label for that dataset, as presented in Table 3 [See Annexure I].

The rest of the unlabeled dataset is labelled by applying the same process. Therefore, twelve classes of registration algorithm strategies were created: $\left\{\left(\mathrm{An}, \mathrm{O}_{\mathrm{k}}\right), \mathrm{n}=1,2,3 \ldots, \mathrm{r}, \mathrm{k}=1,2,3 \ldots, \mathrm{m}\right.$. $\}$, the twelve classes produced from that three registration algorithms and each registration algorithm has four optimization parameters strategy. Finally, the outcomes of the labelling process are the labelled datasetsbased registration algorithms and optimization algorithm ( $\left.\mathrm{An}, \mathrm{O}_{\mathrm{k}}\right)$. The labelled dataset that delivered from the first step is considered a training dataset. The training dataset is then ready for the next stage, which is creating a learning model using a multilayer perceptron classifier (MLP).

\section{Labeling Model}

The second step is creating a learning modal. To construct a learning modal, where the labelled dataset mapped with an MLP classifier to classify them into twelve class as represented in Figures 6 \& 7 [See Annexure I]. 
Testing unlabeled dataset is the third step, where 100 unlabeled datasets were used with a learning modal to classify them.

As described above, registration algorithm selection is considered a classification problem. This problem can be solved by training a classifier to discriminate among the twelve classes, as shown in Table 1, and the learned classifier is then evaluated using a test dataset (unlabeled dataset).

For this purpose, an MLP classifier was selected to learn using a labelled dataset that was created during the first step, as presented in Figure 8 [See Annexure I]. The chosen classifier used 100 labelled datasets: $75 \%$ for training and $25 \%$ for testing.

As illustrated in Figure 8, the outcome of this stage is a learned model, as represented in figure 9.

\section{Registration Strategy Selection}

The final stage is the selection of the best registration algorithm strategy and the best optimization strategy based on the learned model, as displayed in Figure 9 [See Annexure I].

The learned model created during the last stage was used for labelling the test dataset. The primary purpose of the learned model is to assign a label to the unknown input datasets. When the test datasets, which are 100 unlabeled datasets, are mapped to the learned model, the classifier matches the test datasets with the dataset-points, and the best-match result is selected as a label for the unknown dataset. The output of the learned model is thus the labelled dataset, and that label represents the registration algorithm strategy and optimization strategy, which are one of the twelve candidates. As a result, the best registration algorithm and optimization strategy for the unknown dataset has been selected, as described in Figure 9. The last stage is mapping the dataset to the chosen registration strategy and three other registration algorithms to accomplish the registration process.

As stated in Figure 10 [See Annexure I], the registration process included the selected registration algorithm (proposed system), and the other three registration algorithms were chosen randomly to compare their performance. From the results, we can conclude that the registration algorithm chosen is outperforming overall different registration algorithms for all input datasets. The second comparing is between the proposed system and the that delivered from the previous work (old system).

As shown in Figure 11 [See Annexure I], which is comparing the two approaches, the results from comparing are manifest that the second approach, which is select the best registration strategy based on (An, Ok,) produced results better than the old approach, which is select the best registration algorithm based on $(\mathrm{An})$.

\section{Roulette Wheel Selection Method}

The output of the learned model is regarded as the labelled dataset, and that label represents the registration algorithm and optimization strategy (Аn, Ок), which are one of the twelve candidates. As a result, the best registration algorithm and optimization technique for the unknown dataset can be selected, as described in Figure 9. To create the proposed method more reliable, accurate, and robust, the roulette wheel approach is used to select the best registration strategy (RS) from a set of candidates.

There are many methods of how to select the best registration algorithm, such as roulette wheel, rank, and steady-state selection[24]. In the roulette wheel system, which is the most straightforward selection method, all the selected candidates, in accordance with the value of their fitness, or performance, are placed on the roulette wheel. A segment of the roulette wheel, in proportion to that candidate's fitness value, is allocated to everyone where a greater-sized segment is assigned to those with a higher fitness or 
performance value. Subsequently, the virtual roulette wheel is spun and whoever corresponds to the algorithm occupying the segment where the wheel ends are chosen, and the procedure is repetitive till the selection of the required number of individuals is complete [24]. In this method, the fitness of the candidates must be measured. A few individuals of a dataset are used to measure the accuracy (fitness) of all candidates, including the best registration strategy (RS) that is produced from a learned model.

Let us consider $\mathrm{N}$ candidates, each described by its strength $f i>0(\mathrm{i}=1,2, \ldots, \mathrm{N})$. Therefore, the choice likelihood of the $\mathrm{i}^{\text {th }}$ individual is given as in Eq. (3).

$P i=f i / \sum_{i=1}^{N}(f i),(\mathrm{i}=1,2, ., ., \mathrm{N})$

Then, the number of candidates must be determined. From Table 2, this number can be seen to be twelve candidates. A set of experiments was conducted to determine the performances of all twelve candidates. Consequently, each dataset examined all candidates, and the respective output is the accuracy. Moreover, the same procedure applied to all datasets; in this case, it was applied to three datasets. Finally, the fitness values were revealed as the performance (accuracy), as shown in Table 4.

The total amount of times the roulette wheel is rolled is the same as the magnitude of the algorithms. Therefore, it appears from the used method, the wheel is currently separated, each time the wheel ends, the stronger entities have a greater probability of being nominated. MATLAB software was used to run the roulette wheel selection algorithm and the results supported the learned model selection for all datasets as listed in Table 4. Therefore, the novel method makes the proposed system more reliable, robust, and accurate than the system in the previous work.

\section{Discussion}

As previously mentioned, it is essential to locate a universal medical image registration strategy that can present the best outcome for every input dataset. A new system for the development of a multimodal medical image registration technique is presented in this essay. This system chooses from a set of registration algorithms for numerous input datasets, the most widely accepted of such algorithms. The proposed system is called an intelligent selection registration strategy (ISRS) and uses a supervised machine learning strategy and a roulette wheel method to select the best registration strategy (RS). The defined multimodal registration algorithm selection problem was considered in this work. The actuality that the special registration algorithm does not supply the best performance for every dataset forms the footstone of the problem statement. Consequently, it is essential to find the most active registration algorithm that is appropriate for resolving this problem instead of developing new registration algorithms. As such, the machine learning and roulette wheel technique formed the foundation of the recommended strategy. The benchmark dataset was mapped to three registration algorithms in order to generate a labelling dataset and an MLP classifier as a learning paradigm to test the datasets to assess the learned model.

Two factors govern the new technique submitted in this paper, namely an optimization algorithm and a registration algorithm, which were utilized as labels for unlabeled datasets. The comparison procedure demonstrated that all of the other registration algorithms in every dataset are surpassed by the new technique (Figure 10) [See Annexure I]. The combination of the optimization algorithm and the registration algorithm having a critical influence on the ultimate performance of the chosen registration algorithm strategy forms the cornerstone of this argument. Two techniques formed the second comparison, which was between the former system that is uniquely dependent on labels and the basis of randomly chosen registration algorithms and the new technique in which the labels were based on the optimization algorithm and the registration algorithm (Figure 11) [See Annexure I]. Furthermore, the 
results verify the excellent performance of the new technique with every input dataset in comparison with the other systems.

The recommended technique, labelling based on optimization strategy and registration algorithms, surpasses the other procedures, which are exclusively based on the registration algorithms (Figure 12) [See Annexure I]. Consequently, a vital function is occupied by the optimization strategy in enhancing the performance of the registration algorithm. To increase the intelligence of the proposed system, a new registration strategy was established, the machine learning and roulette wheel selection method, that represents the registration algorithms and optimization strategy (Аn, Ок). Several experiments were then conducted, the results of which show that the novel approach leads to a considerably faster, reliable, accurate, and more robust registration strategy.

\section{Conclusion and Future Work}

The main achievement of this paper is introducing a novel method for achieving multimodal medical image registration based on manipulating the complementary and competitive nature of the algorithmic approaches behind a wide spectrum of registration approaches. A comprehensive investigation of a wide range of registration algorithms is conducted for the purpose of understanding and quantifying their registration capabilities as well as influence of their control parameters. Consequently, a supervised randomized machine learning strategy is proposed for selecting the best registration algorithm for a given registration instance, and for determining the optimal control parameters for such algorithm. Several experiments have been conducted to verify the capabilities of the proposed selection strategy with respect to registration reliability, accuracy, and robustness. The findings delivered from comparing the results of the novel method and the previous approach are more accurate, robust and reliable. The outcomes of this work proof that the change or tunning the control parameter (optimization methods) and refining the final selection using one of the selection methods such as the roulette wheel method can provide the best selection for the registration algorithm. This opens new horizons for researchers to attain better findings.

\section{References}

[1] N. Andrade, F. A. Faria, and F. A. M. Cappabianco, "A Practical Review on Medical Image Registration: From Rigid to Deep Learning-Based Approaches," Proc. - 31st Conf. Graph. Patterns Images, SIBGRAPI 2018, pp. 463-470, 2019, doi: 10.1109/SIBGRAPI.2018.00066.

[2] F. S. Bashiri, A. Baghaie, R. Rostami, Z. Yu, and R. M. D'Souza, "Multi-modal medical image registration with full or partial data: A manifold learning approach," J. Imaging, vol. 5, no. 1, pp. 12-17, 2019, doi: 10.3390/jimaging5010005.

[3] Y. Hu et al., "Weakly-supervised convolutional neural networks for multimodal image registration," Med. Image Anal., vol. 49, pp. 1-13, 2018, doi: 10.1016/j.media.2018.07.002.

[4] K. K. Brock, S. Mutic, T. R. McNutt, H. Li, and M. L. Kessler, "Use of image registration and fusion algorithms and techniques in radiotherapy: Report of the AAPM Radiation Therapy Committee Task Group No. 132: Report," Med. Phys., vol. 44, no. 7, pp. e43-e76, 2017, doi: 10.1002/mp.12256.

[5] Y. Qing, M. Li, Q. Wei, and X. Ren, "A Registration Method for Multimodal Medical Images Using Contours Mutual Information,” Int. J. Adv. Res. Artif. Intell., vol. 3, no. 4, pp. 3-7, 2014, doi: 10.14569/ijarai.2014.030401.

[6] C. Venkateswara, K. M. M. Rao, A. S. Manjunath, and R. V. N. Srinivas, "Optimization of Automatic Image Registration Algorithms and Characterization."

[7] D. Wujanz, L. Barazzetti, M. Previtali, and M. Scaioni, "A COMPARATIVE STUDY among THREE REGISTRATION ALGORITHMS: PERFORMANCE, QUALITY ASSURANCE and ACCURACY," ISPRS Ann. Photogramm. Remote Sens. Spat. Inf. Sci., vol. 42, no. 2/W9, pp. 779-786, 2019, doi: 
10.5194/isprs-archives-XLII-2-W9-779-2019.

[8] S. Nag, "Image Registration Techniques: A Survey," 2017, doi: 10.17605/OSF.IO/RV65C.

[9] A. S. Lundervold and A. Lundervold, "An overview of deep learning in medical imaging focusing on MRI,” Z. Med. Phys., vol. 29, no. 2, pp. 102-127, 2019, doi: 10.1016/j.zemedi.2018.11.002.

[10] G. Song, J. Han, Y. Zhao, Z. Wang, and H. Du, "A Review on Medical Image Registration as an Optimization Problem," Curr. Med. Imaging Rev., vol. 13, no. 3, pp. 274-283, 2017, doi: $10.2174 / 1573405612666160920123955$.

[11] E. Irmak and M. Burak TÜRKÖZ, "A Useful Implementation of Medical Image Registration for Brain Tumor Growth Investigation in a Three Dimensional Manner," IJCSNS Int. J. Comput. Sci. Netw. Secur., vol. 17, no. 6, pp. 155-161, 2017.

[12] S. Y. Guan, T. M. Wang, C. Meng, and J. C. Wang, "A review of point feature based medical image registration," Chinese J. Mech. Eng. (English Ed., vol. 31, no. 4, 2018, doi: 10.1186/s10033-018-0275-9.

[13] B. T. T. Yeo, "Learning Task-Optimal Image Registration with Applications in Localizing Structure and Function in the Cerebral Cortex," Cereb. Cortex, no. 2002, p. 141, 2010.

[14] O. A. Omer and M. Abdel-Nasser, "C16. Multimodal medical image registration approach using an artificial immune system for noisy and partial data," Natl. Radio Sci. Conf. NRSC, Proc., vol. 2013-Janua, no. April, pp. 266-273, 2013, doi: 10.1109/NRSC.2013.6587923.

[15] A. Davari, T. Lindenberger, A. Häberle, V. Christlein, A. Maier, and C. Riess, "Image Registration for the Alignment of Digitized Historical Documents," pp. 1-39, 2017.

[16] S. Mambo, K. Djouani, Y. Hamam, B. Van Wyk, and P. Siarry, “Techniques,” vol. 12, no. 1, pp. 48-55, 2018.

[17] J. B. A. Maintz and M. a Viergever, "An Overview of Medical Image Registration Methods (Cited by: 2654)," Nature, vol. 12, no. 6, pp. 1-22, 1996, doi: 10.1.1.39.4417.

[18] F. P. M. Oliveira and J. M. R. S. Tavares, "Medical image registration: A review," Comput. Methods Biomech. Biomed. Engin., vol. 17, no. 2, pp. 73-93, 2014, doi: 10.1080/10255842.2012.670855.

[19] J. Ofverstedt, J. Lindblad, and N. Sladoje, "Fast and Robust Symmetric Image Registration Based on Distances Combining Intensity and Spatial Information," IEEE Trans. Image Process., vol. 28, no. 7, pp. 3584-3597, 2019, doi: 10.1109/TIP.2019.2899947.

[20] D. Sigurdson, V. Bulitko, S. Koenig, C. Hernandez, and W. Yeoh, "Automatic Algorithm Selection In Multi-agent Pathfinding,” 2019.

[21] P. Sharma, A. Wadhwa, and K. Komal, "Analysis of Selection Schemes for Solving an Optimization Problem in Genetic Algorithm," Int. J. Comput. Appl., vol. 93, no. 11, pp. 1-3, 2014, doi: 10.5120/162565714.

[22] J. Rice, “The Algorithm Selection Problem - Abstract Models,” pp. 75-152, 1974.

[23] H. Elkeshreu, O. Basir, “Algorithm Selection in Multimodal Medical Image Registration-12.”vol. 97, no. 11, pp. 1-3, 2020, doi: 10.5120/16256-5714.

[24] N. Jankowski and M. Grochowski, "Comparison of instances seletion algorithms I. algorithms survey," Lect. Notes Artif. Intell. (Subseries Lect. Notes Comput. Sci., vol. 3070, pp. 598-603, 2004, doi: 10.1007/978-3-540-24844-6_90. 


\section{Annexure I Tables \& Figures}

Table 1

Principles for categorizing registration techniques

\begin{tabular}{|c|c|c|c|c|c|}
\hline Dimensionality & $\begin{array}{c}\text { Registration } \\
\text { Basis }\end{array}$ & Interaction & Modalities & Subject & Object \\
\hline 2D-to-2D & $\begin{array}{c}\text { Feature-based } \\
\text { Approach }\end{array}$ & Manual Interactive & Single-Modal & $\begin{array}{c}\text { Intra- } \\
\text { subject }\end{array}$ & Brain \\
\hline 3D-to-3D & $\begin{array}{c}\text { Intensity-based } \\
\text { Approach }\end{array}$ & Semi-Interactive & Multimodal & $\begin{array}{c}\text { Inter- } \\
\text { Subject }\end{array}$ & Eye \\
\hline 2D-t0-3D & & Auto interactive & & $\begin{array}{c}\text { Atlas- } \\
\text { Subject }\end{array}$ & Lungs \\
\hline
\end{tabular}

Table 2

Medical Image Categorization[23]

\begin{tabular}{|c|c|}
\hline Anatomical Structure & Functional Structure \\
\hline $\begin{array}{c}\text { Magnetic Resonance } \\
\text { Image (MRI) }\end{array}$ & $\begin{array}{c}\text { Electrical Impedance } \\
\text { Tomography (EIT) }\end{array}$ \\
\hline $\begin{array}{c}\text { Computer } \\
\text { Tomography (CT) }\end{array}$ & $\begin{array}{c}\text { Single Photon Emission } \\
\text { Computed Tomography } \\
\text { (SPECT }\end{array}$ \\
\hline X-Ray & $\begin{array}{c}\text { Positron Emission } \\
\text { Tomography (PET) }\end{array}$ \\
\hline Ultrasound & $\begin{array}{c}\text { Electroencephalography } \\
\text { (EEG) }\end{array}$ \\
\hline
\end{tabular}

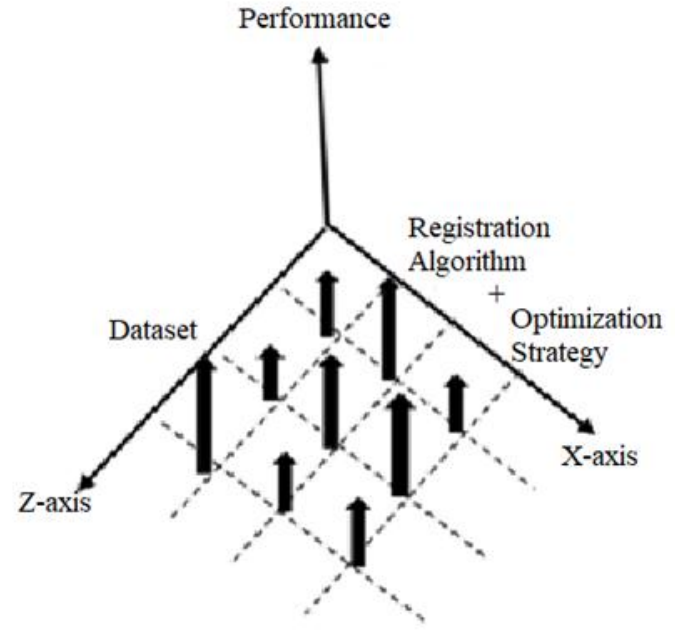

Fig. 1. Algorithm selection problem in three-dimensional space. 


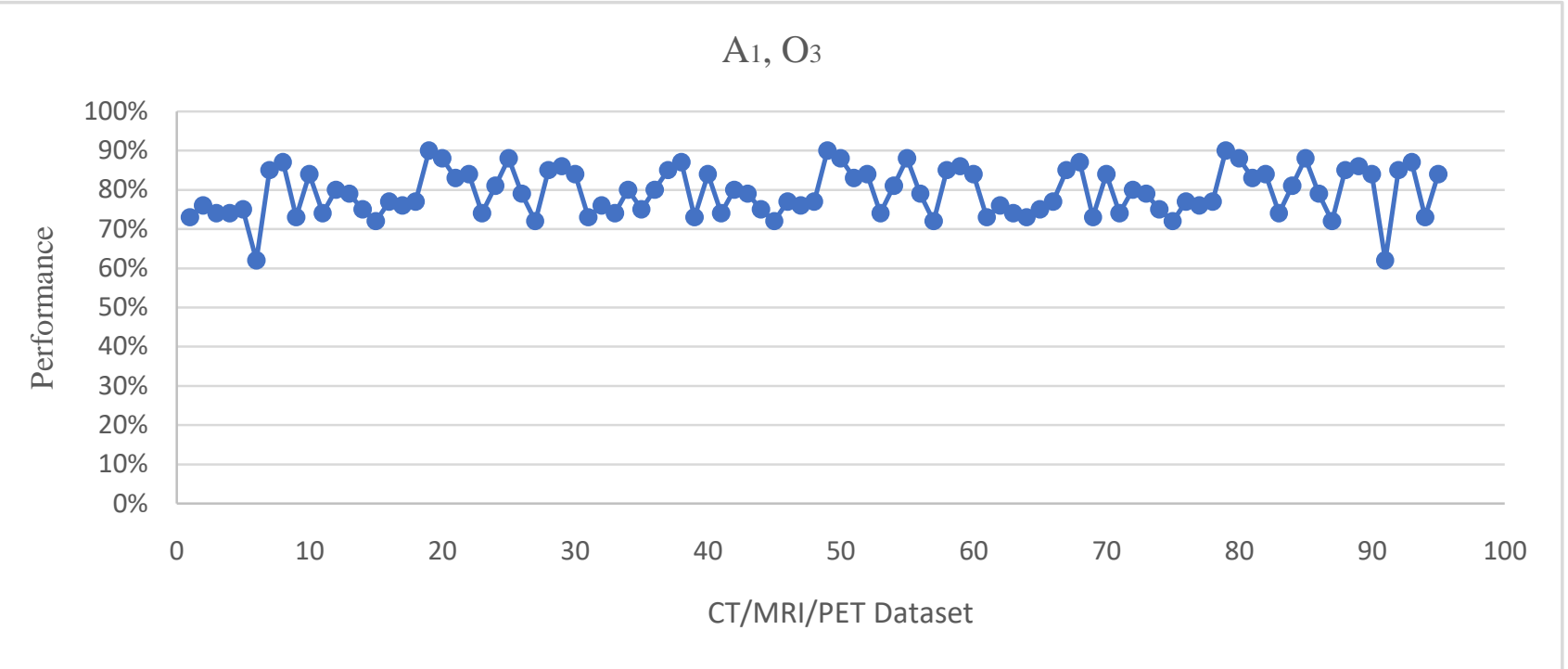

Fig. 2. The relationship between datasets and registration algorithm A1 with optimization strategy O3.

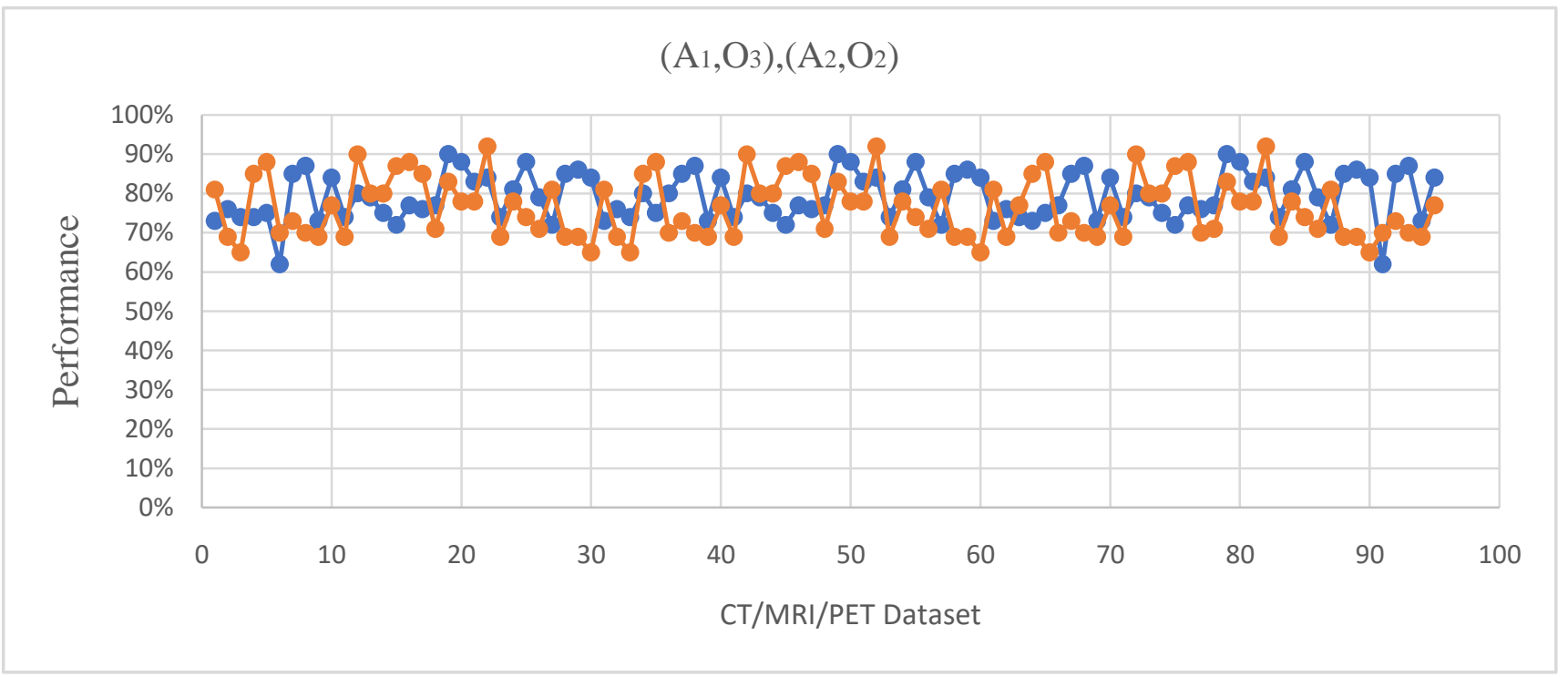

Fig. 3. The relationship between datasets and registration algorithms $\mathrm{A} 1$ and $\mathrm{A} 2$ with different optimization strategy 


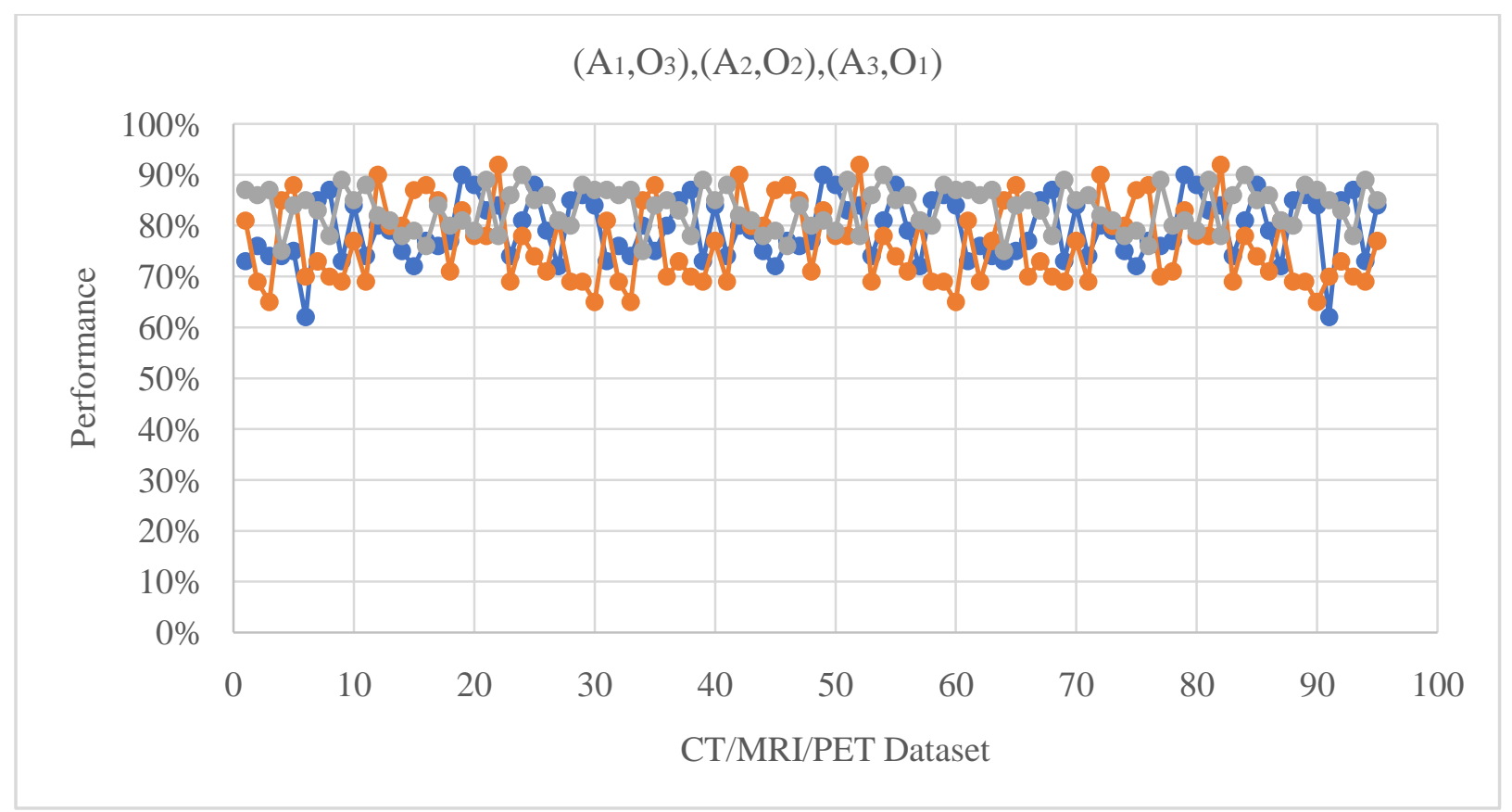

Fig. 4. The relationship between datasets and registration algorithms A1, A2, A3 with different optimization strategy

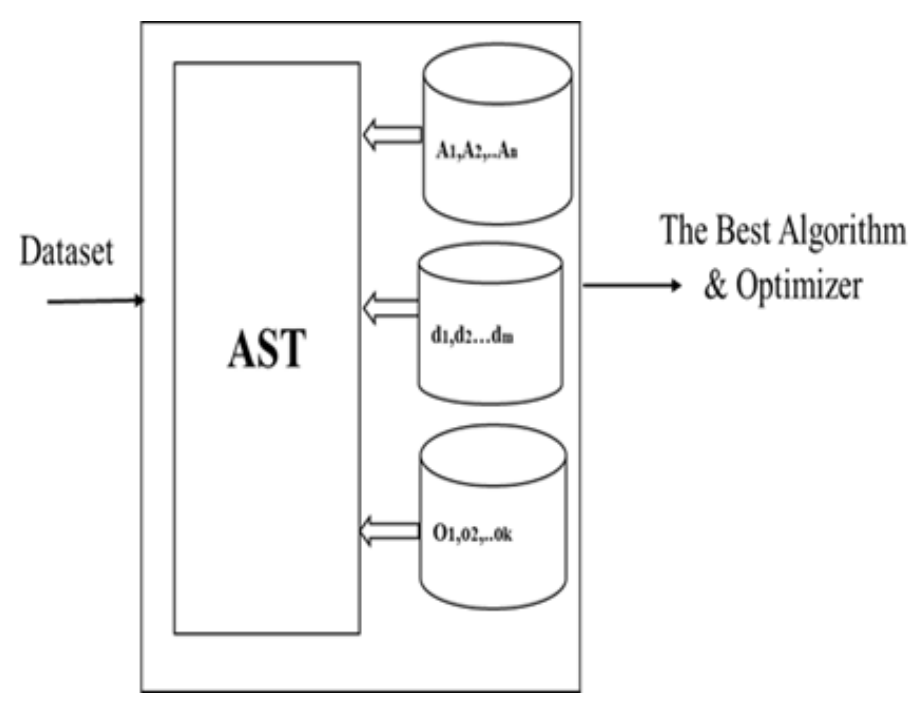

Fig. 5. The problem solution 
Table 3

Dataset labelling based on registration algorithm and optimization strategy parameters

\begin{tabular}{|c|c|c|c|c|c|c|c|c|c|c|c|c|c|}
\hline \multirow{2}{*}{ Dataset } & \multicolumn{5}{|c|}{$\mathrm{RS}_{1 \%}$} & \multicolumn{5}{c|}{$\mathrm{RS}_{2 \%}$} & \multicolumn{5}{c|}{$\mathrm{RS}_{3 \%}$} & Labels \\
\cline { 2 - 14 } & $\mathrm{O}_{1}$ & $\mathrm{O}_{2}$ & $\mathrm{O}_{3}$ & $\mathrm{O}_{4}$ & $\mathrm{O}_{1}$ & $\mathrm{O}_{2}$ & $\mathrm{O}_{3}$ & $\mathrm{O}_{4}$ & $\mathrm{O}_{1}$ & $\mathrm{O}_{2}$ & $\mathrm{O}_{3}$ & $\mathrm{O}_{4}$ & \\
\hline 1 & 80 & 92 & 88 & 85 & 87 & 82 & 89 & 93 & 84 & 89 & 85 & 95 & $\mathrm{RS}(3,4)$ \\
\hline 2 & 88 & 85 & 95 & 90 & 87 & 76 & 86 & 93 & 85 & 93 & 87 & 89 & $\mathrm{RS}(1,3)$ \\
\hline. &. &. &. &. &. &. &. &. &. &. &. &. &. \\
\hline. &. &. &. &. &. &. &. &. &. &. &. &. &. \\
\hline. &. &. &. &. &. &. &. &. &. &. &. &. &. \\
\hline. &. &. &. &. &. &. &. &. &. &. &. &. &. \\
\hline 179 & 93 & 87 & 85 & 86 & 84 & 90 & 88 & 91 & 89 & 85 & 87 & 88 & $\mathrm{RS}(1,1)$ \\
\hline
\end{tabular}

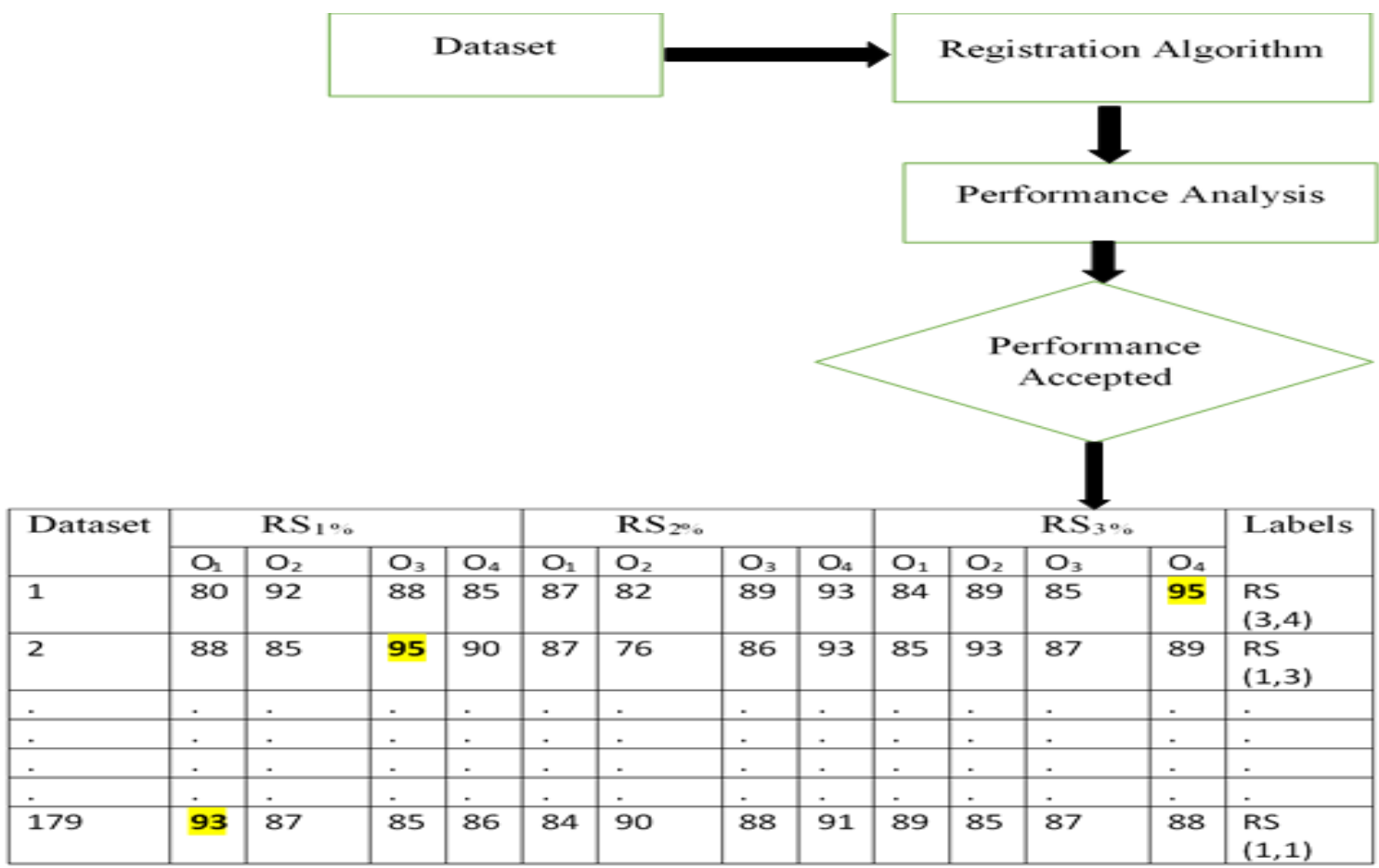

Fig. 6. Dataset labelling. 


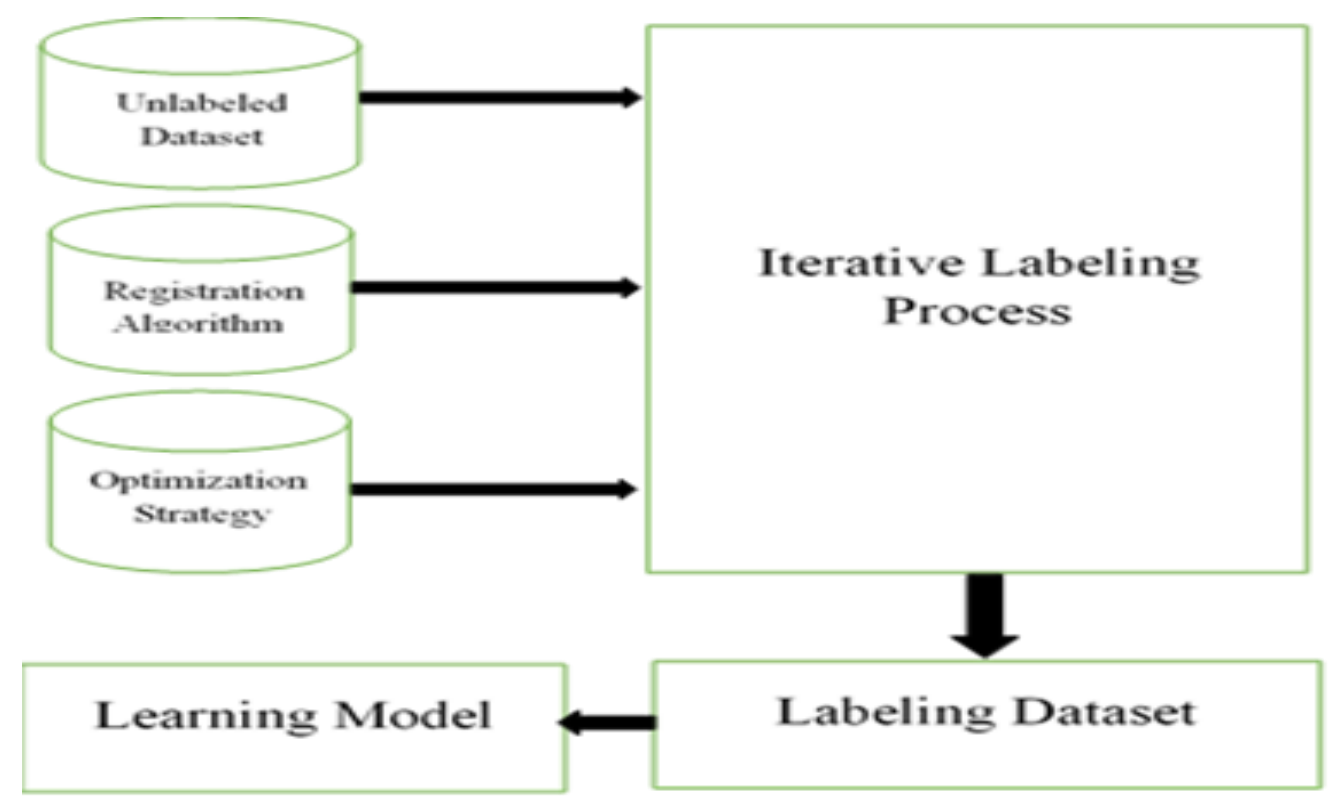

Fig. 7. Schematic diagram of the novel method.

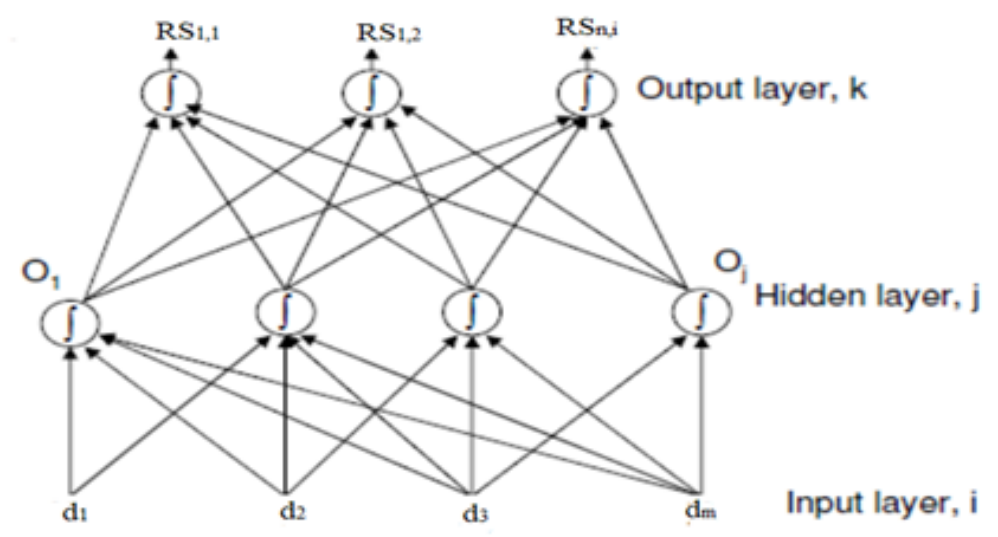

Fig. 8. Multilayer perceptron classifier (MLP) 


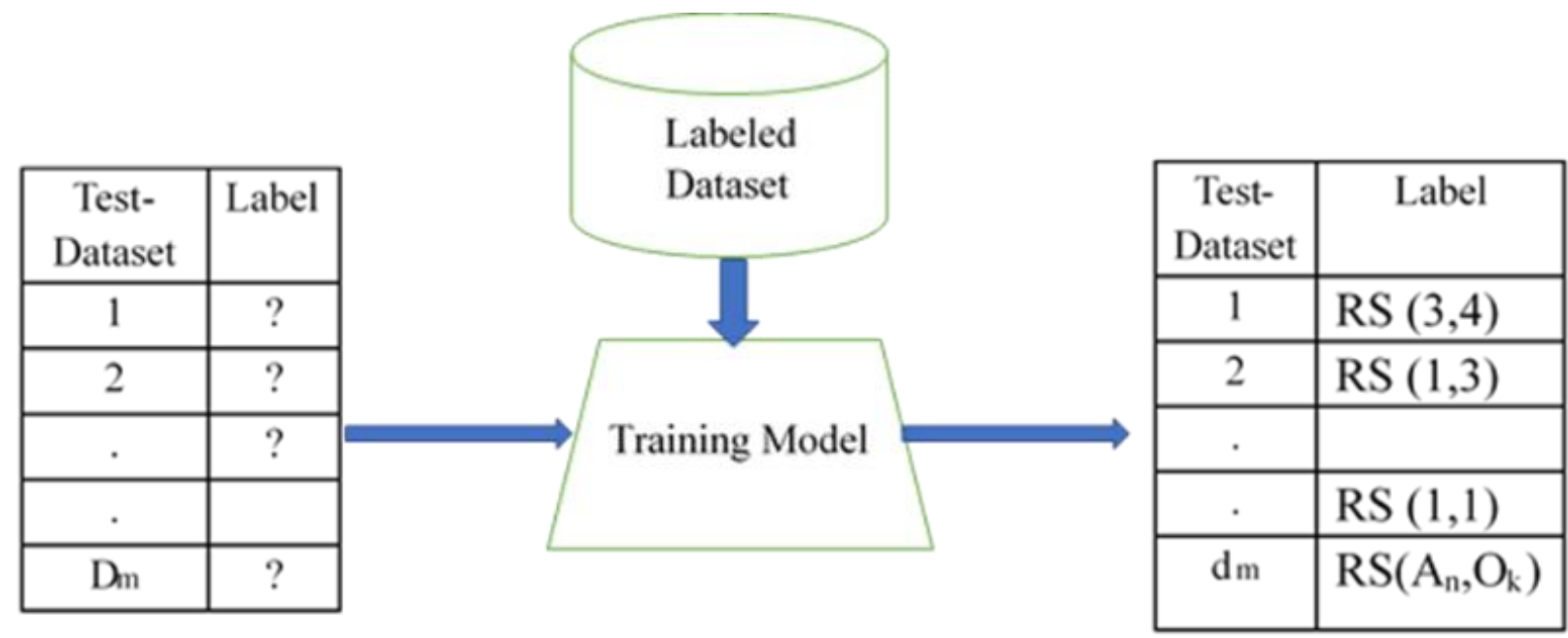

Fig. 9. Registration algorithm selection

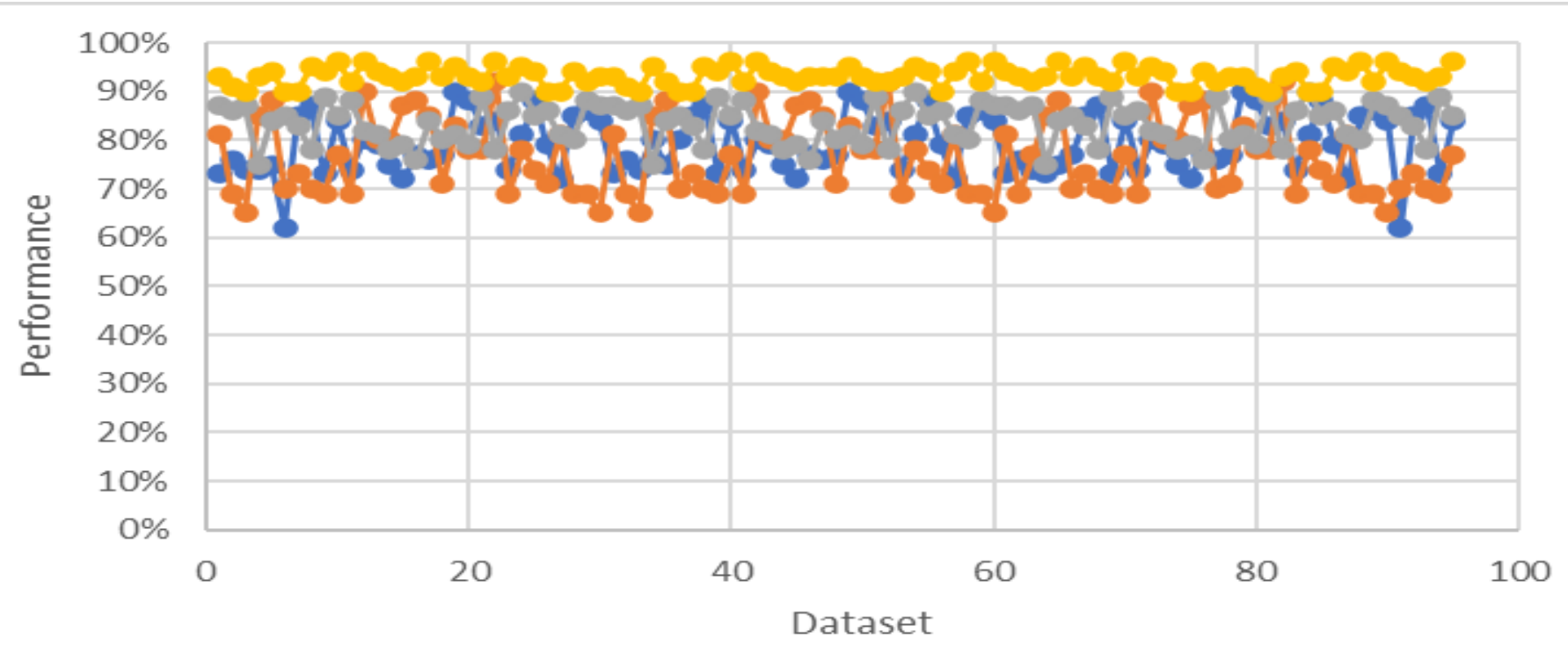

$\longrightarrow \mathrm{A} 1, \mathrm{O} 3 \longrightarrow \mathrm{A} 2, \mathrm{O} 2 \longrightarrow \mathrm{A} 3, \mathrm{O} 1 \longrightarrow$ Proposed System(An,Ok)

Fig. 10. System Performance: Learning Dataset 


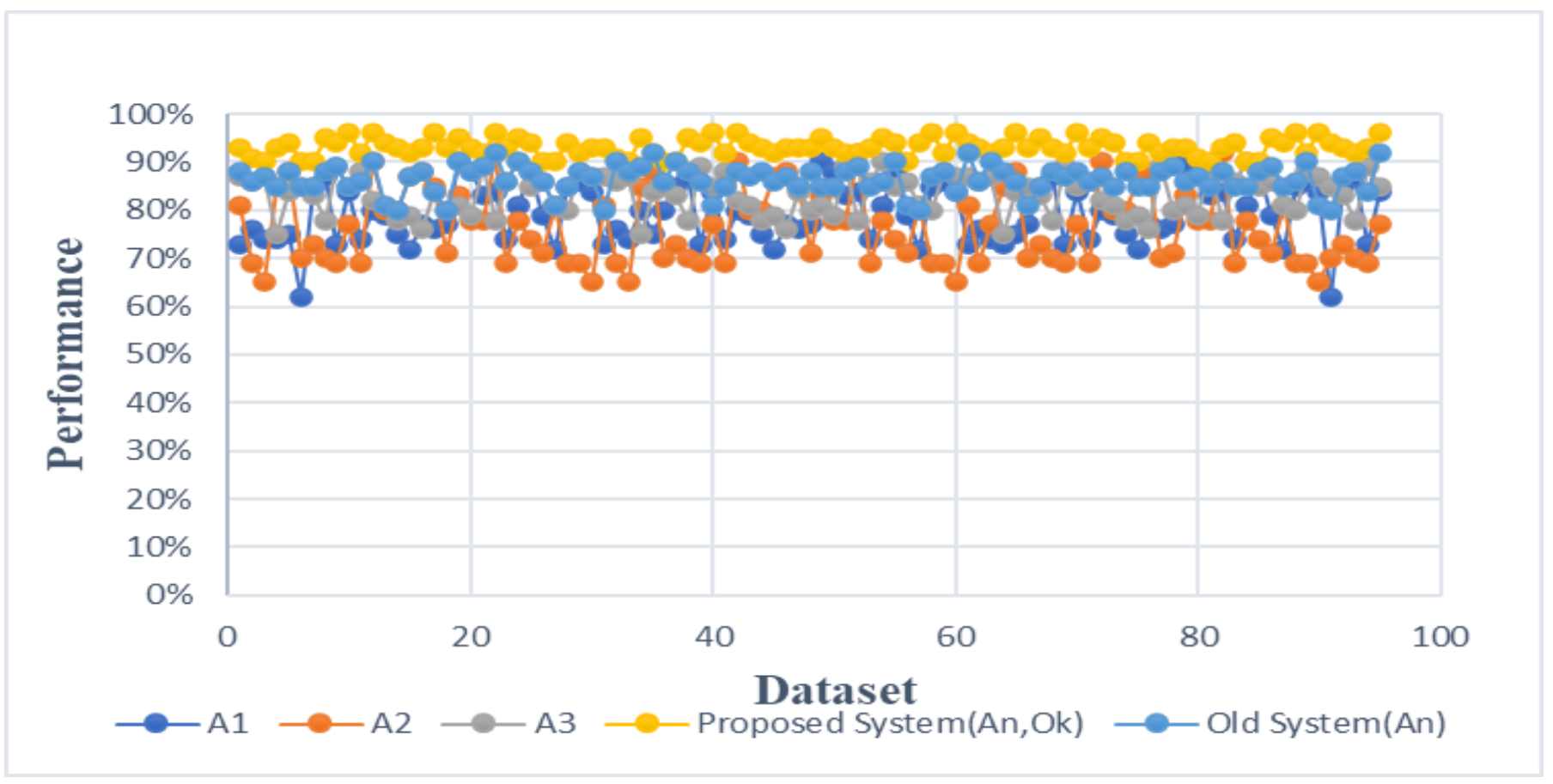

Fig. 11. System Performance: Unlearned Dataset

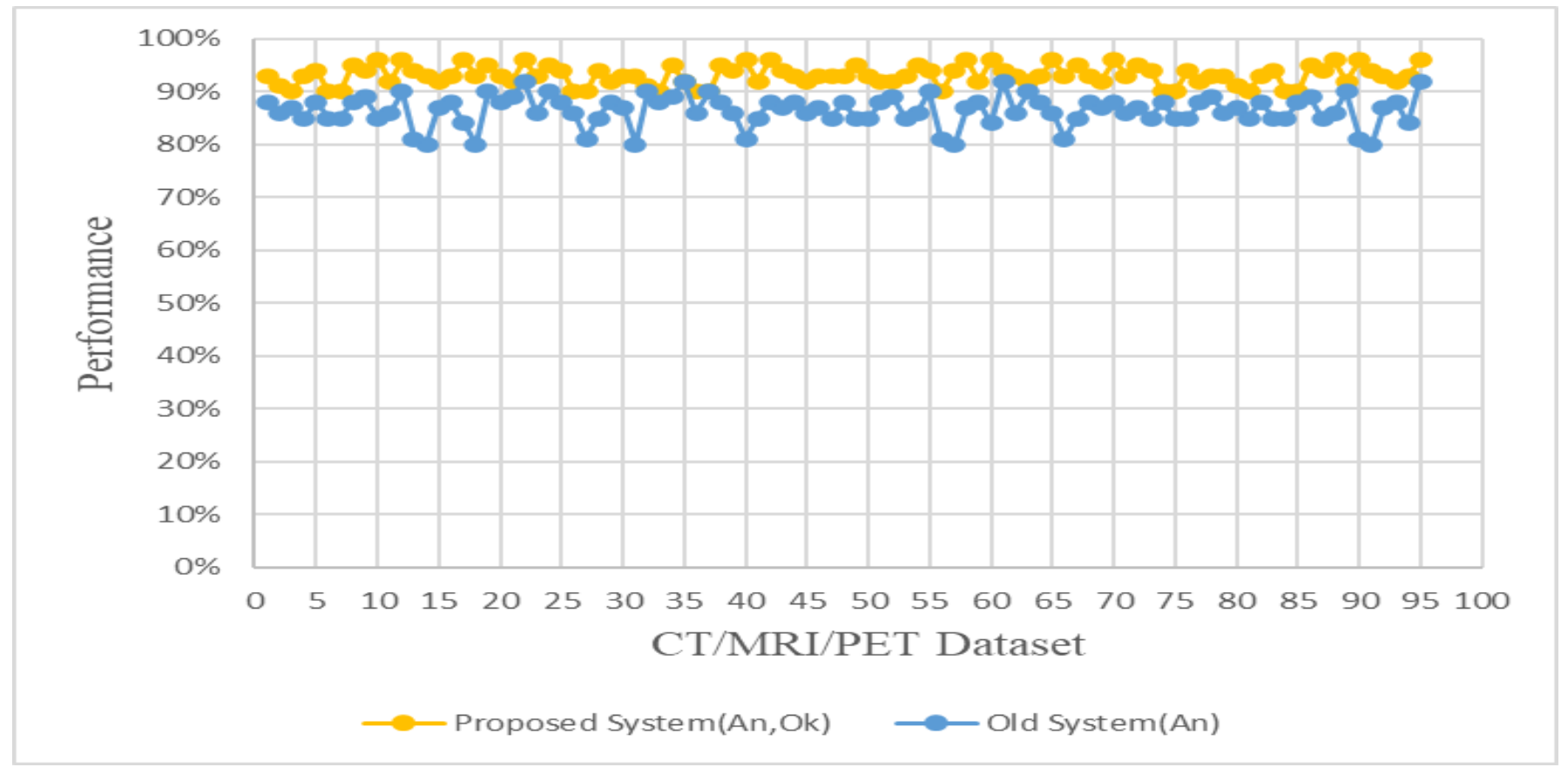

Fig. 12. Comparison of a method with optimization parameters and without optimization parameters 
Table 4

The best performances of all candidates

\begin{tabular}{|c|c|c|c|c|}
\hline $\mathrm{A}_{1}, \mathrm{O}_{3}$ & $\mathrm{~A}_{2}, \mathrm{O}_{2}$ & $\mathrm{~A}_{3}, \mathrm{O}_{1}$ & $\begin{array}{c}\text { Proposed } \\
\left.\text { System (An, } \mathrm{O}_{k}\right)\end{array}$ & $\begin{array}{c}\text { Old } \\
\text { System (An) }\end{array}$ \\
\hline $73 \%$ & $81 \%$ & $87 \%$ & $93 \%$ & $88 \%$ \\
\hline $76 \%$ & $69 \%$ & $86 \%$ & $91 \%$ & $86 \%$ \\
\hline $74 \%$ & $65 \%$ & $87 \%$ & $90 \%$ & $87 \%$ \\
\hline $74 \%$ & $85 \%$ & $75 \%$ & $93 \%$ & $85 \%$ \\
\hline $75 \%$ & $88 \%$ & $84 \%$ & $94 \%$ & $88 \%$ \\
\hline $62 \%$ & $70 \%$ & $85 \%$ & $90 \%$ & $85 \%$ \\
\hline $85 \%$ & $73 \%$ & $83 \%$ & $90 \%$ & $85 \%$ \\
\hline $87 \%$ & $70 \%$ & $78 \%$ & $95 \%$ & $88 \%$ \\
\hline $73 \%$ & $69 \%$ & $89 \%$ & $94 \%$ & $89 \%$ \\
\hline $84 \%$ & $77 \%$ & $85 \%$ & $96 \%$ & $85 \%$ \\
\hline $74 \%$ & $69 \%$ & $88 \%$ & $92 \%$ & $86 \%$ \\
\hline $80 \%$ & $90 \%$ & $82 \%$ & $96 \%$ & $90 \%$ \\
\hline $79 \%$ & $80 \%$ & $81 \%$ & $94 \%$ & $81 \%$ \\
\hline $75 \%$ & $80 \%$ & $78 \%$ & $93 \%$ & $80 \%$ \\
\hline & & & & \\
\hline
\end{tabular}

\section{A1,03 A2,O2 A3,01 - Proposed System(An,Ok) $\quad$ Old System(An)}

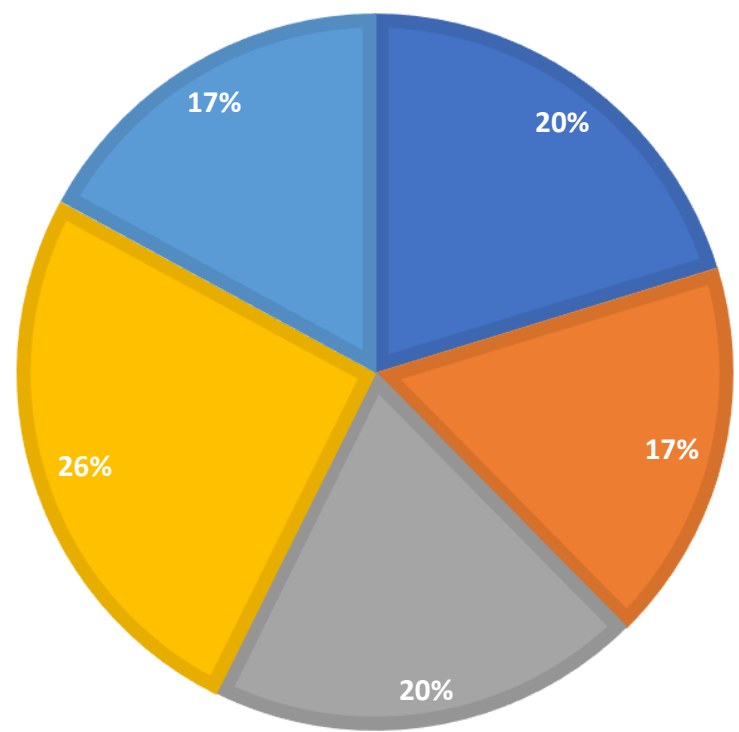

Fig. 13. The roulette wheel selection fitness of four registration strategies (RS) 